\title{
Escala de Avaliação do Transtorno Dismórfico Corporal (EA-TDC): Propriedades Psicométricas
}

\author{
Assessment Scale for Body Dysmorphic Disorder (AS-BDD): \\ Psychometric Properties
}

\author{
Kátia Perez Ramos* \& Elisa Medici Pizão Yoshida \\ Pontificia Universidade Católica de Campinas, Campinas, Brasil
}

\begin{abstract}
Resumo
Este estudo analisou as propriedades psicométricas da segunda versão da Escala de Avaliação do Transtorno Dismórfico Corporal - EA-TDC, de auto-relato, 40 itens, desenvolvidos para avaliar três critérios do Transtorno Dismórfico Corporal caracterizado por: preocupação excessiva com um imaginado defeito na aparência; sofrimento clinicamente significativo e prejuízo funcional em campos importantes da vida; a preocupação não é melhor explicada por outro transtorno. A análise de construto por juízes indicou acordo sobre 35 itens. Esses foram respondidos por 30 pacientes diagnosticados com TDC e 400 universitários. A validade de critério indicou capacidade de discriminação dos itens entre os indivíduos dos dois grupos amostrais. A análise fatorial exploratória sugeriu estrutura unidimensional da escala que permite medir, "preocupação excessiva e sofrimento com um imaginado defeito e os prejuízos sobre o funcionamento em diferentes áreas da vida". Os itens retidos revelaram excelente consistência interna. Definição de pontos de cortes e da capacidade de discriminar outros transtornos devem ser pesquisados.
\end{abstract}

Palavras-chave: Avaliação psicológica, auto-relato, assistência à saúde, transtorno somatoforme.

\begin{abstract}
This study analyzed the psychometric properties of the second version of the Assessment Scale for Body Dysmorphic Disorder (AS-BDD). It is a scale of self-report, with 40 items, developed to evaluate 3 dimensions of Body Dysmorphic Disorder (BDD): (a) excessive concern with a minor or imagined flaw in his/ her personal physical characteristics, (b) significant clinical suffering and functional damage in important areas of life, and (c) such concern is not better explained by another disorder. The construct analysis by judges indicated accordance in 35 items which were answered by 30 patients diagnosed with BDD and 400 university students. The validity of criterion measures indicated ability to discriminate the items among the individuals of both sampled groups. The exploratory factor analysis suggested a unidimensional structure of the scale which allows to measuring "excessive concern with a minor or imagined flaw in his/her personal physical characteristics and the functional damages in different areas of life". The withheld items have shown excellent internal consistency. Cut point definitions and the ability to discriminate other disorders should be further studied.

Keywords: Psychological assessment, self-report, health care, somatoform disorder.
\end{abstract}

O transtorno dismórfico corporal é um novo nome para uma velha desordem (Warwick, 1995). Tem sido descrito nas literaturas européia e japonesa por uma variedade de expressões, sendo a mais comum dismorfofobia, termo que teria sido utilizado por Morselli, pela primeira vez, em 1886 (Phillips \& Kaye, 2007). Na classificação psiquiátrica americana é encontrada primeiramente no DSM-III (American Psychiatric Association [APA], 1980), como um exemplo de transtorno somato-

*Endereço para correspondência: Centro de Ciências da Vida, Pontifícia Universidade Católica de Campinas, Curso de Pós-Graduação em Psicologia, Rua John Boyd Dunlop, s/n, Jardim Ipaussurama, Campinas, SP, Brasil 13060-904. E-mail: eyoshida.tln@terra.com.bre katiapramos@hotmail.com forme sem nenhum critério diagnóstico, o que só aparece no DSM-III-R (APA, 1987). Segundo o DSM-III-R, o transtorno dismórfico corporal é caracterizado pela preocupação com um imaginado defeito na aparência. Se uma ligeira anomalia física está presente, a preocupação do indivíduo é acentuadamente excessiva e causa sofrimento clinicamente significativo e prejuízo no funcionamento social, ocupacional e em outros campos importantes de sua vida. Na revisão mais recente do DSM, designada DSM-IV-TR ${ }^{\mathrm{TM}}$ (APA, 2000), poucas informações foram acrescidas ao texto sobre o TDC. Nas características diagnósticas, foi incluída uma referência à musculatura como possível foco de preocupação dos pacientes, enquanto nas características associadas foram incluídos exercícios excessivos (p.ex. muscu- 
lação), dietas e trocas freqüentes de roupas. O diagnóstico diferencial foi complementado com a seguinte frase: "A prática excessiva de exercícios (p.ex., musculação) que pode ocorrer neste transtorno para corrigir uma falha percebida na aparência deve ser diferenciada dos exercícios saudáveis e do excesso de exercícios que pode ocorrer como parte de Transtorno da Alimentação" (APA, 2000, p. 490).

De acordo com Phillips e Kaye (2007) os sintomas que não são específicos do TDC podem ser encontrados em diversos transtornos psiquiátricos, como na depressão (Phillips, Didie, \& Menard, 2007), na psicose (Phillips, 2004; Phillips, Menard, Pagano, Fay, \& Stout, 2006), no transtorno obsessivo compulsivo (TOC; Drummond, Pillay, Benson, \& Jones, 2008; Neziroglu \& Tobias, 1993; Phillips \& Kaye, 2007; Phillips et al., 2006), em transtornos de personalidade (Jakubietz, Jakubietz, Kloss, Joerg, \& Gruenert, 2007), e na anorexia nervosa (Buhlmann, Teachman, Gerbershagen, Kikul, \& Rief, 2008; Phillips \& Kaye, 2007). Em relação às queixas associadas ao TDC envolvem, em geral, falhas imaginárias ou leves na face ou na cabeça (como acne, cicatrizes, rugas, inchaço, assimetria ou pêlos faciais excessivos). Outras preocupações comuns incluem tamanho, forma ou algum outro aspecto do nariz, da boca, dos olhos, das pálpebras, das sobrancelhas, das orelhas, da boca, dos dentes, da mandíbula, do queixo, das bochechas ou da cabeça e odores corporais desagradáveis (Phillips et al., 2006; Rosen, 1997; Sarwer, Wadden, Pertschuk, \& Whitaker, 1998). Entretanto, qualquer outra parte do corpo pode ser o foco de preocupação - por exemplo, genitais, abdômen, nádegas, quadris, ombros (Bowe, Leyden, Crerand, Sarwer, \& Margolis, 2007). Embora a queixa seja freqüentemente específica, pode ser, por vezes, vaga, e alguns indivíduos evitam descrever os seus defeitos em detalhes podendo se referir à sua "feiúra" em geral (Buhlmann et al., 2008).

Preocupações culturais acerca da aparência e da importância da apresentação física adequada podem influenciar ou ampliar preocupações sobre a imaginada deformidade física de pessoas que reúnem carcaterísticas predisponentes para o TDC (Moriyama, 2003). Isto vale tanto para homens quanto para mulheres, uma vez que evidências preliminares sugerem que o TDC acomete igualmente ambos os sexos (Jakubietz et al., 2007; Warwick, 1995). A primeira manifestação de sintomas é, em geral, entre o início da adolescência e 20 anos, (APA, 2000), com taxas de prevalência entre $0,7 \%$ e $2,2 \%$ da população geral (Jakubietz et al., 2007). Em clínicas de cirurgia plástica este percentual cresce para $6 \%$ a $15 \%$ (APA, 2000).

Os indivíduos com TDC freqüentemente buscam e recebem tratamentos médicos gerais, dentários ou cirúrgicos com o objetivo de corrigir os seus defeitos imaginados (Drummond et al., 2008; Sarwer \& Crerand, 2008; Sobanski \& Schimidtz, 2000). Além disso, sentem vergonha de falar sobre suas preocupações e quando chegam a procurar serviços psicológicos já desen- volveram muitos outros problemas, característicos de outras classificações psiquiátricas, como fobia social, TOC, depressão, entre outros (Sarwer \& Crerand, 2008). Em contextos nos quais são executados procedimentos com fins cosméticos, o TDC é insuficientemente reconhecido (Buhlmann et al., 2008), e este tipo de tratamento médico pode levar a uma intensificação dos sintomas ou a novas preocupações, que por sua vez ensejam a busca de procedimentos adicionais desnecessários, com o aumento de insatisfação por parte do portador (Dufresne, Phillips, Vittorio, \& Wilkel, 2001). Segundo Jakubietz et al. (2007) aproximadamente $5 \%$ das pessoas que procuram por cirurgia plástica são portadoras de TDC, e o tratamento correto seria o psicológico e não o cirúrgico. No entanto, essa população só tardiamente é encaminhada às clínicas e consultórios de psicólogos pela solicitação da família ou por um médico mais atento ao transtorno (Amaral, 2001; Rabinowitz, Neziroglu, \& Roberts, 2007). $\mathrm{E}$, finalmente, cabe mencionar que a dificuldade ou inabilidade em detectar o TDC, pode levar os profissionais que lidam com esta população a se tornar alvo de processos e/ou agressões físicas, pela realização de cirurgias e tratamentos desnecessários (Buhlmann et al., 2008; Rohrich, 2000).

\section{Instrumentos de Avaliação do TDC}

No que se refere aos instrumentos de avaliação do TDC, tem-se escasso material. A maioria foi desenvolvida para a avaliação de transtornos cujos sintomas guardam alguma relação com os do TDC, como os transtornos alimentares, TOC, entre outros. Por exemplo, um dos primeiros instrumentos foi o Body Shape Questionnaire (BSQ; Cooper, Taylor, Cooper, \& Fairburn, 1987). Composto por 34 itens, avalia a insatisfação com o tamanho ou forma corporal no desenvolvimento, manutenção e tratamento da anorexia e bulimia, mas não avalia diversos aspectos do TDC, como por exemplo, os comportamentos de checagem e camuflagem da parte do corpo em relação à qual a pessoa se sente insatisfeita, seus pensamentos e obsessões e esquiva de eventos sociais.

O Multidimensional Body-Self Relations Questionnaire (MBSRQ; Brown, Cash, \& Mikulka, 1990) contém 60 itens que avaliam a atitude das pessoas em relação à sua imagem corporal ou aparência física. Possui 10 subescalas, sendo as mais importantes para a avaliação do TDC, a subescala Satisfação com Áreas do Corpo, que mensura a insatisfação do paciente com áreas específicas de sua aparência; a subescala Avaliação da Aparência, que analisa os sentimentos sobre atratividade física e, por fim; a Orientação da Aparência, que avalia a tendência para prestar atenção ou desenvolver o comportamento de se arrumar ou esconder determinada parte do corpo, de forma exagerada. É indicada para a avaliação geral da imagem corporal e também como ferramenta para os diagnósticos de TDC, transtornos alimentares (anorexia e bulimia) e obesidade mórbida. 
Outras escalas foram criadas com o objetivo de avaliar aspectos da aparência física, tais como, a BDS - Body Dissatisfaction Scale (Gardner, 1991), mais especificamente relacionada ao diagnóstico da bulimia e anorexia e a OIS - Overvalued Ideas Scale (Neziroglu \& Tobias, 1993), voltada à avaliação específica do pensamento obsessivo sobre o defeito, não englobando, portanto, outros comportamentos que fazem parte do diagnóstico do TDC. Phillips, McElroy, Keck, Pope e Hudson (1993) modificaram a Y-BOCBS - Yale-Brown Obsessive Compulsive Scale (Goodman et al., 1989), com o propósito de mensurar o TDC. Para tanto, propuseram a BDDYBOCS - Yale-Brown Obsessive Compulsive Scale modified by Body Dysmorphic Disorder. Além de outras modificações, um quadro relativo aos "pensamentos sobre o defeito do corpo" foi inserido para englobar a ocorrência de pensamentos sobre o defeito e o esforço feito pelo indivíduo para controlá-los, mas se restringe a investigar a sintomatologia relacionada à obsessão e compulsão nos pacientes com TDC. Os pacientes com o transtorno, que foram submetidos à psicoterapia, tiveram sua pontuação diminuída na escala (Phillips et al., 1993), entretanto nenhum estudo psicométrico do instrumento foi realizado.

Rosen e Reiter (1996) avaliaram os instrumentos existentes sobre imagem corporal, construídos até o ano de 1996, e concluíram que nenhum englobava todas as características necessárias para o diagnóstico do TDC, sendo que em sua maioria eram utilizados para a avaliação e diagnóstico de transtornos alimentares. A partir desse levantamento, propuseram um instrumento específico para o diagnóstico do TDC, denominado BDDE (Body Dysmorphic Disorder Examination) e baseado nos critérios do DSM-IV (APA, 1994). A BDDE é uma entrevista clínica semi-estruturada constituida por 34 questões que avaliam a presença e a severidade de um sintoma característico do TDC segundo seis áreas: preocupação com e avaliação negativa da aparência; autoconsciência, desconforto e sentimento de ser observado quando em público; excessiva importância à aparência na auto-avaliação; evitação de situações sociais ou de atividades em público ou evitação de contato físico com outros; camuflagem da aparência com roupas, maquiagem ou postura corporal, e; comportamento de auto-observação, em forma de auto-inspeção, arrumar-se repetidamente, reasseguramento e se comparar a outras pessoas. As propriedades psicométricas foram avaliadas com base em pacientes de clínica para avaliação e tratamento do TDC e outras queixas relacionadas à auto-imagem $(n=259)$, divididos em pacientes com TDC e não-TDC (não é indicado o número de sujeitos de cada sub-grupo); e um grupo controle de universitários $(n=295)$ e funcionários da universidade $(n=140)$. A BBDE revelou consistência interna adequada para os quatro grupos amostrais (alphas entre 0,81 e 0,93$)$, boa precisão de teste e reteste, com intervalo de duas semanas para os pacientes $(0,94)$ e para os univesitários e funcionários reunidos $(0,87)$. Para esses dois grupos, obteve-se bom acordo entre juízes $(0,86$ e 0,99$)$. Medidas de validade concorrente, em que foram estimadas as correlações entre a BDDE e várias medidas de imagem corporal e ajustamento psicológico (Multidimensional Body-Self Relations Questionnaire [MBSRQ]; Body Shape Questionnaire [BSQ]; Brief Symptom Inventory; e Rosenberg Self-Steem Scale), apontaram altas correlações entre a escala e as medidas de imagem corporal negativa, sintomas psicológicos mais severos e baixa auto-estima. O estudo de validade de critério, que comparou os grupos clínicos com os não-clínicos, mostrou que os sujeitos com diagnóstico de TDC apresentavam escores significantemente mais elevados que os dos demais três grupos e que os sujeitos do grupo clínico, sem transtorno de TDC, apresentavam escores mais elevados que os dos sub-grupos não clínico. E, finalmente, os universitários reportavam mais sintomas que os funcionários da universidade. Uma análise de regressão logística hierárquica testou a contribuição independente da BDDE para predizer membros do grupo clínico e não clínico depois do controle estatístico de outros preditores (idade, sintomas psicológicos, auto-estima, e três subescalas do MBSRQ que mediam aspectos da imagem corporal). Mostraram-se preditores do estatus clínico, a idade, a auto-estima, uma sub-escala do MBSRQ (Escala de Orientação da Aparência) e a BDDE. Ademais, os resultados indicaram que a informação das outras três medidas de imagem corporal eram redundantes com a BDDE, e que ela era melhor preditor do estatus clínico. Também foi estimada a validade preditiva da BDDE, comparando seus resultados com diagnósticos de TDC realizados por psiquiatras, após duas semanas. O coeficiente kappa de Cohen, indicando o acordo entre as duas avaliações foi de 0,82 .

Dufresne et al. (2001) desenvolveram um breve questionário para o diagnóstico do TDC, na prática dermatológica, o BDDQ-DV - Body Dysmorphic Disorder Questionnaire - Dermatology Version. Para tanto, utilizaram como referência o BDDQ - Body Dysmorphic Disorder Questionnaire (Phillips, Atala, \& Pope, 1995 citados por Dufresne et al., 2001), construido para ser aplicado a pacientes psiquiátricos, e também baseado no DSM-IV (APA, 1994). No processo de adaptação do BDDQ para a clínica dermatológica (BDDQ - DV), as respostas em escala Likert de cinco pontos foram substituidas por respostas sim / não. Entretanto duas questões se mantiveram com Likert de cinco pontos, pois mediam a intensidade do sentimento de angústia em relação ao defeito imaginário e dos prejuízos na área social, ocupacional e em outras áreas importantes da vida do indivíduo. O BDDQ-DV, foi aplicado a 46 pacientes de uma clínica de cirurgia plástica com finalidade estética. Todos responderam ao instrumento e foram avaliados por cirurgião, cego para as respostas ao BDDQ-DV, com uma entrevista clínica semi-estruturada baseada nos critérios do DSM-IV para avaliar o TDC. Sete pacientes foram diagnosticados por ele como apresentando TDC. 
Quanto ao BDDQ-DV, nove pacientes pontuaram positivamente para TDC, sendo que desses, em três casos a avaliação do cirurgião não corroborou o diagnóstico. Com base nos resultados, estabeleceu-se que o instrumento apresentava $100 \%$ de sensibilidade e $92,3 \%$ de especificidade. O valor de predição positiva teria sido de $70 \% \mathrm{e}$ o valor preditivo negativo de $100 \%$. Dos três pacientes que não apresentavam sintomas de TDC de acordo com o cirurgião, um veio a apresentá-los no período pós-operatório, o que aumentou a especificidade para $94,7 \%$. A pesquisa traz ainda uma estimativa de precisão entre avaliadores, concernente à severidade dos defeitos identificados pelos itens avaliados com a escala Likert. O Coeficiente de Correlação Intraclasses foi de 0,88.

Considerando que os portadores de TDC buscam usualmente, em primeiro lugar, profissionais ligados a procedimentos corretores estéticos e só tardiamente psicólogos ou psiquiatras, Ramos (2004) desenvolveu a Escala de Avaliação do TDC (EA-TDC), para profissionais da área da saúde. Seu objetivo foi o de contribuir para a identificação precoce deste transtorno e evitar procedimentos caros e desnecessários, que podem por em risco a saúde do portador do distúrbio e também causar problemas aos próprios profissionais, que têm os resultados de seus tratamentos questionados, pelas insatisfações permanentes destes pacientes. Os itens da escala foram gerados tomando por base três instrumentos de avaliação do TDC já existentes (BDD-YBOCS, BDDE, BDDQ-DV) e os critérios diagnósticos do DSM-IV-TR ${ }^{\mathrm{TM}}$ (APA, 2000). Foram agrupados segundo as seguintes dimensões teóricas: (a) preocupação com um imaginado defeito na aparência. Se uma ligeira anomalia física está presente, a preocupação do indivíduo é acentuadamente excessiva; (b) a preocupação causa sofrimento clinicamente significativo e prejuízo no funcionamento social, ocupacional e em outros campos importantes de sua vida; (c) a preocupação não é melhor explicada por outros transtornos como a anorexia. Foram construídos 50 itens avaliados em relação à pertinência, objetividade, clareza e relevância e descartados dez itens. A pertinência dos 40 itens foi analisada, de forma independente, por psiquiatras e psicólogos $(N=5)$, sendo que mais cinco itens foram descartados por não atingirem concordância de no mínimo $80 \%$. A Escala com 35 itens foi então aplicada em 15 pacientes de cirurgia plástica estética diagnosticados clinicamente como portadores de TDC e 15 sem o diagnóstico de TDC. A prova $U$ de Mann-Whitney mostrou que a Escala é sensível para discriminar os indivíduos dos dois grupos. A precisão de teste-reteste (20 dias) indicou a estabilidade das avaliações nos dois grupos ( $r=0,93$ e $r=0,95)$. O instrumento ficou então composto por 35 itens medidos por respostas Likert de quatro pontos (concordo plenamente, concordo, discordo, discordo plenamente). Entretanto a pesquisa apresentava algumas limitações, tais como: o limitado número de participantes $(N=30)$ e o fato de ter poucos itens relacionados ao diagnóstico diferencial. Além dis- so, não permitia avaliar a intensidade do transtorno. Por exemplo, o item 13. "Busco freqüentemente a opinião de outras pessoas de que a(s) parte(s) do meu corpo da(s) qual(is) não gosto não é(são) tão anormal(is) ou defeituosa(s) quanto eu penso ser". Se o sujeito assinalar que discorda ou discorda plenamente não é possível saber se "não busca a opinião de outras pessoas" ou se discorda de possuir "uma parte do corpo tão anormal ou defeituosa", ou ainda se não concorda com o fato de que a "parte do corpo da qual não gosta não é tão anormal quanto ele pensa ser." A presente pesquisa teve, portanto, como objetivo a reformulação da Escala de Avaliação do Transtorno Dismórfico Corporal - EA-TDC para profissionais da área da saúde (Ramos, 2004) e a verificação de novas evidências de suas propriedades psicométricas. As modificações realizadas deram origem à segunda versão da EA-TDC.

\section{Método}

\section{Participantes}

A amostra foi integrada por dois grupos de participantes: G1 -30 portadores de TDC, avaliados pela primeira autora com base em entrevista e segundo os critérios do DSM-IV-TR ${ }^{\mathrm{TM}}$ (APA, 2000), sendo: 70\% mulheres, com idade média de 32,46 anos ( $D P=10,62$, mín. 18 e máx. de 54 anos), 36,5\% tinham companheiro, 83,5\% tinham ao menos ensino superior incompleto e renda mensal média de $\mathrm{R} \$ 1.678,33$ (mín. $\mathrm{R} \$ 800,00$ e máx. $\mathrm{R} \$ 5.500,00)$; G2 - 400 estudantes universitários de instituição particular de ensino superior (sendo, 196 do curso de psicologia, 98 de engenharia e 106 de educação física), dos quais: 74,5\% mulheres, com idade média de 26,5 anos $(D P=7,43$, mín. 18 e máx. de 51 anos), $74,75 \%$ tinham companheiro e renda mensal média de $\mathrm{R} \$ 1.735,00$ (mín. $\mathrm{R} \$ 500,00$ e máx. $\mathrm{R} \$ 10.000,00$ ). Apesar de muito distintos quanto ao tamanho, ambos os grupos eram comparáveis quanto ao sexo (predomínio de mulheres: $\mathrm{G} 1=70 \%$ e $\mathrm{G} 2=74,5 \%$ ); apresentavam maioria de pessoas entre 18 e 30 anos $(\mathrm{G} 1=46,5 \%$ e $\mathrm{G} 2=$ $79 \%)$; com escolaridade superior incompleta $(\mathrm{G} 1=$ $73,5 \%$ e $\mathrm{G} 2=97,75 \%$ ). Eles se diferenciam quanto à proporção de participantes com companheiros (menor no $\mathrm{G} 1,36,5 \%$ x G2=74,75\%) e em relação à faixa de renda, ligeiramente mais alta no G1 $(53,5 \%$ dos participantes possuem renda de $\mathrm{R} \$ 1.100,00$ a $\mathrm{R} \$ 2.000,00$, e no G2 48\% dos partici-pantes possuem renda de $\mathrm{R} \$ 801,00$ a $\mathrm{R} \$ 1.100,00)$. Isto, provavelmente se deve ao fato dos participantes do G2 serem estudantes universitários, ainda dependentes dos pais.

\section{Juizes}

Para a análise de construto (Pasquali, 1999), realizada para verificar a adequação da representação comportamental das características do TDC expresso em cada item, foi composto um grupo (G3) de dez profissionais 
das áreas de Psicologia $(N=4)$, Psiquiatria $(N=2)$, Cirurgia Plástica $(N=2)$ e Odontologia $(N=2)$, com tempo de formação profissional entre 8 e 31 anos. Eles indicaram pacientes (atuais e passados), possivelmente portadores de TDC, para a composição de G1 e também colaboraram como juízes para a estimativa da validade de conteúdo.

\section{Instrumentos}

Escala de Avaliação do Transtorno Dismórfico Corporal (EA-TDC; Ramos, 2009). Para a composição da segunda versão da EA-TDC, de auto-relato, substituiuse as repostas originais em escala Likert (que variavam de 1. não concordo a 4. concordo plenamente), por afirmativas que permitissem a classificação da intensidade dos sintomas. Para cada item foram desenvolvidas quatro alternativas, como por exemplo: Item 1: 0 - Estou satisfeito com a minha aparência física, 1 - Estou insatisfeito com a minha aparência física, 2 - Estou muito insatisfeito com minha aparência física, 3 - Estou tão insatisfeito com minha aparência física que não posso suportar. Além disso, aos 35 itens originais da Escala (Ramos, 2004) foram acrescidos cinco itens $(4,17,22,25$ e 32), destinados a avaliar sintomas dos transtornos alimentares como anorexia e bulimia e dos transtornos de gênero, de acordo com o DSM-IV-TR ${ }^{\mathrm{TM}}$ (APA, 2000), visando possibilitar um diagnóstico diferencial. Com isso, a Escala submetida à verificação das propriedades psicométricas ficou composta por 40 itens que tinham, respectivamente, como objetivo avaliar: (1) Satisfação com a aparência física; (2) Realização de tratamentos cosméticos e estéticos; (3) Percepção de um defeito físico; (4) Comportamento de comer compulsivo; (5) Esquiva de atividade física; (6) Desempenho no trabalho e aparência física; (7) Pensamentos sobre o defeito; (8) Ansiedade produzida pelo olhar dos outros; (9) Comparação de si mesmo com outras pessoas em revistas e TV; (10) Incômodo por uma parte do corpo; (11) Auto-avaliação negativa devido ao defeito; (12) Ser tratado diferentemente por outros, devido ao defeito na aparência; (13) Perceber a anormalidade ou o exagero da preocupação com o defeito; (14) Busca de reafirmação sobre o defeito; (15) Esquiva de situações sociais; (16) Freqüência de olhar no espelho; (17) Preocupação com ganho de peso; (18) Realização de cirurgia plástica estética; (19) Interferência social devido o defeito; (20) Preocupação com peso corporal; (21) Preocupação com o defeito em situações sociais; (22) Ansiedade por se alimentar; (23) Esquiva de contato sexual; (24) Tempo gasto com camuflagem; (25) Satisfação com opção sexual; (26) Esquiva de olhar o defeito; (27) Preocupação com a aparência física no geral; (28) Esquiva de contato físico como beijo e abraço; (29) Comparação da aparência física com outras pessoas ao redor; (30) Avaliação negativa por outros devido ao defeito; (31) Uso de camuflagem; (32) Sentimento sobre o peso corporal; (33) Alteração de postura corporal; (34) Vergonha sobre o defeito na aparência; (35) Perda de interesse em atividades diárias; (36) Esquiva de situações públicas, de sair de casa; (37) Repetição de atividades diárias; (38) Desempenho geral e o defeito na aparência; (39) Nível de atratividade física percebida; e (40) Realização de dietas alimentares.

Roteiro A. Desenvolvido para orientar os participantes do G3 a identificar possíveis portadores de TDC, entre os seus pacientes. Descrevia os comportamentos característicos do TDC; a procura por tratamentos estéticos; os problemas que podem ser causados aos profissionais quando da realização de tratamentos desnecessários; a manutenção ou piora do TDC como resultado desses tratamentos.

Roteiro B. Desenvolvido para orientar os participantes do G3 a estabelecer o contato com seus pacientes, provavelmente portadores de TDC, e convidá-los a participar da pesquisa. Enfatizava que se devia evitar o uso da expressão transtorno dismórfico corporal, além de destacar a relevância da possível participação na pesquisa.

Roteiro C. Desenvolvido para a identificação de características do transtorno dismórfico corporal de acordo com o DSM-IV-TR ${ }^{\mathrm{TM}}$ (APA, 2000), foi utilizado em entrevistas com os pacientes encaminhados pelos participantes do G3, com vistas à composição do G1 e também respondido pelos participantes do G2. Incluía questões sobre a preocupação com alguma parte do corpo; a freqüência dos pensamentos sobre a parte do corpo da qual o indivíduo não gostava e se havia algum sentimento de angústia como conseqüência desses pensamentos; se já havia se submetido a cirurgia plástica estética e, se havia ficado satisfeito(a) com os resultados da cirurgia plástica estética. Além disso, foi investigada a interferência no funcionamento social, ocupacional, familiar ou em outras funções diárias do indivíduo por conseqüência de sua preocupação com a aparência física.

Ficha de Análise Teórica dos Itens. Desenvolvida para a categorização dos itens pelos juízes com base no conceito do TDC presente no DSM-IV-TR ${ }^{\mathrm{TM}}$ (APA, 2000): (a) preocupação com um imaginado defeito na aparência. Se uma ligeira anomalia física está presente, a preocupação do indivíduo é acentuadamente excessiva; (b) a preocupação causa sofrimento clinicamente significativo e prejuízo no funcionamento social, ocupacional e em outros campos importantes de sua vida; (c) a preocupação não é melhor explicada por outros transtornos como a anorexia. Cada item deveria ser associado a apenas um fator.

\section{Procedimento}

Contataram-se cirurgiões plásticos, dentistas, psicólogos e psiquiatras para solicitar-lhes que indicassem pacientes, provavelmente portadores de TDC. Para tanto, foram esclarecidos quanto aos principais indicadores do transtorno. Estes profissionais entraram em contato com seus pacientes (atuais ou passados) e convidaram-nos a participar da pesquisa. Foram contatados 56 pacientes no total, sendo que 41 aceitaram participar e foram encaminhados para entrevista individual com a primeira autora. 
Desses, 30 participantes se encaixaram nos critérios do DSM-IV-TR ${ }^{\mathrm{TM}}$ (APA, 2000) para o TDC e foram consultados novamente quanto à disposição para participar da pesquisa, quando assinaram o Termo de Consentimento Livre e Esclarecido e responderam à segunda versão da EA-TDC.

Os participantes de G2 foram testados coletivamente em sala de aula. Após uma breve explicação sobre os objetivos da pesquisa e sobre o caráter voluntário da participação, era solicitada a assinatura do Termo de Consentimento Livre e Esclarecido, aos que estivessem de acordo. A seguir, os participantes liam e respondiam às perguntas do Roteiro C e, só então, recebiam os formulá- rios da EA-TDC ( $2^{\mathrm{a}}$ versão). Antecedendo a aplicação desse instrumento, dois exemplos eram dados com o objetivo de instruí-los sobre a forma correta de responder aos itens. O projeto de Pesquisa foi aprovado pelo Comitê de Pesquisa com Seres Humanos da Instituição em que a pesquisa foi desenvolvida (Processo $\mathrm{n}^{\circ}$. 612-08).

\section{Resultados e Discussão}

Em relação às questões do Roteiro $\mathrm{C}$ que pretendiam identificar características do TDC entre os participantes do G1 e G2, a Tabela 1 apresenta a distribuição dos participantes (2006).

Tabela 1

Questões do Roteiro C e Distribuição dos Participantes de G1 e G2 quanto às Respostas

\begin{tabular}{|c|c|c|c|c|c|}
\hline \multirow[t]{2}{*}{ Questão } & & \multicolumn{2}{|c|}{ G $1(n=30)$} & \multicolumn{2}{|c|}{ G $2(n=400)$} \\
\hline & & $F$ & $\%$ & $F$ & $\%$ \\
\hline \multirow{3}{*}{$\begin{array}{l}\text { Você se preocupa muito com alguma parte de seu } \\
\text { corpo [se sente insatisfeito(a) e/ou acha feio(a)]? }\end{array}$} & Sim & 30 & 100 & 323 & 8 \\
\hline & Não & - & - & 68 & 92 \\
\hline & Total & 30 & 100 & 400 & 100 \\
\hline \multirow{3}{*}{$\begin{array}{l}\text { Se você respondeu que sim, você pensa muito sobre } \\
\text { essa(s) parte(s) de seu corpo de que não gosta } \\
\text { a ponto desses pensamentos o atormentarem? }\end{array}$} & Sim & 30 & 100 & 1 & 3 \\
\hline & Não & - & - & 31 & 9 \\
\hline & Total & 30 & 100 & 400 & 100 \\
\hline \multirow{5}{*}{$\begin{array}{l}\text { Quantas vezes por dia você pensa na parte do corpo } \\
\text { da qual não gosta? }\end{array}$} & De 10 a 20 vezes & 2 & 7 & 1 & 100 \\
\hline & De 21 a 30 vezes & 5 & 16,5 & - & - \\
\hline & De 31 a 40 vezes & 5 & 6,5 & - & - \\
\hline & Mais de 40 vezes & 18 & 60 & - & - \\
\hline & Total & 30 & 100 & 1 & 100 \\
\hline \multirow{3}{*}{$\begin{array}{l}\text { Essa sua preocupação com a aparência tem interferido } \\
\text { negativamente na escola, faculdade, trabalho } \\
\text { ou em outras funções diárias? }\end{array}$} & Sim & 29 & 97 & 32 & 100 \\
\hline & Não & 1 & 3 & 32 & 100 \\
\hline & Total & 30 & 100 & 32 & 100 \\
\hline \multirow[t]{6}{*}{ Quantas cirurgias plásticas você já realizou? } & 1 cirurgia & 2 & 23 & 24 & 89 \\
\hline & De 2 a 5 & 5 & 55 & 3 & 11 \\
\hline & De 6 a 10 & 1 & 11 & - & - \\
\hline & De 11 a 15 & - & - & - & - \\
\hline & Acima de 15 & 1 & 11 & - & - \\
\hline & Total & 9 & 100 & 27 & 100 \\
\hline \multirow{4}{*}{$\begin{array}{l}\text { Você se sentiu satisfeito(a) com o resultado da(s) } \\
\text { cirurgia(s) ou tratamento(s) estético(s)? }\end{array}$} & Sim & 1 & 89 & 27 & 100 \\
\hline & Não & 8 & 11 & - & - \\
\hline & Total & 9 & 100 & 27 & 100 \\
\hline & Total & 9 & 100 & 27 & 100 \\
\hline
\end{tabular}

Em relação à presença de angústia produzida pelos pensamentos sobre a parte do corpo da qual o sujeito não gosta, os participantes do G1 (100\%) distinguiram-se claramente dos do G2 que declararam não pensar sobre o assunto (Tabela 1). Este é um dado importante para o diagnóstico do TDC, pois evidencia o quanto a preocupação com a aparência física interfere na vida do indivíduo ( $97 \%$ de G1), sendo que a maioria (93\%) chegava a pen- 
sar mais de 20 vezes ao dia no seu "defeito". Em contraste, indivíduos sem este transtorno, negam qualquer interferência nas suas vidas devido a este fator $(100 \%$ de G2; Tabela 1). Segundo Phillips et al. (2006), os portadores de TDC geralmente esquivam-se de sair para lugares públicos como shoppings, bares, cinemas, clubes e supermercados por medo das avaliações negativas que acreditam que possam produzir sobre a sua aparência ou o "defeito", o que acaba resultando em baixo repertório social e prejuízo no funcionamento social. Interessante observar que apesar da preocupação com a aparência física, $70 \%$ dos participantes do G1 nunca chegaram a se submeter a cirurgias plásticas, diferentemente do encontrado em outros estudos, em que cerca de $15 \%$ e $73 \%$ das amostras já havia se submetido a algum tipo de cirurgia reparadora (Buhlmann et al., 2008; Sarwer \& Crerand, 2008). Embora não se tenha dados comparativos, o fato de apenas $30 \%$ dos participantes ter se submetido a alguma cirurgia reparadora é de certa forma surpreendente e provavelmente pode ser explicado por motivos econômicos que tornam proibitivos procedimentos dessa natureza a uma grande parcela da população brasileira. Especialmente, quando se trata de pessoas como as da amostra que tinham renda entre $\mathrm{R} \$ 1.000,00$ e $\mathrm{R} \$ 2.000,00$.

Apesar de eventuais restrições financeiras, deve ser observado que $30 \%$ de G1 haviam se submetido a pelo menos uma cirurgia, contrastando com G2, onde apenas $6 \%$ encontravam-se nesta situação. Sendo que desses últimos, $89 \%$ haviam realizado apenas uma cirurgia, contra 75\% de G1 (Tabela 1). E ainda, é preciso considerar que dentre os participantes de G1 todos referiram insatisfação com os resultados das cirurgias a que haviam se submetido. Estes resultados corroboram, portanto mais uma vez, que estes tratamentos são inócuos nos casos de pessoas com TDC, podendo mesmo levar a uma piora do transtorno e a novas preocupações, com o aumento da insatisfação em relação à própria aparência (Amaral, 2001; Phillips, 1999; Sarwer \& Crerand, 2008).

\section{Análise de Construto}

Dentro da sistemática de construção de um instrumento (Pasquali, 1999), a EA-TDC (2 $2^{\mathrm{a}}$ versão) passou, inicialmente, pela análise de juízes com o objetivo de verificar a adequação dos itens para a avaliação do transtorno dismórfico corporal. Cinco itens obtiveram concordância inferior a $80 \%$ quanto à sua pertinência a um fator (itens: 8, 20, 25, 30 e 38) e, por isso, foram descartados. Os trinta e cinco itens restantes foram então submetidos à avaliação empírica, por meio da estimativa da validade de critério e validade de construto.

\section{Validade de Critério}

A validade de critério foi obtida por meio do método dos grupos comparados (Anastasi \& Urbina, 2000). A Prova $U$ de Mann-Whitney indicou que a maioria dos
35 itens permite diferenciar os participantes de G1 do $\mathrm{G} 2(p<0,05)$, com exceção dos itens 4,22 e $37(p>0,05)$. Esses tinham sido desenvolvidos com o propósito de permitir o diagnóstico diferencial avaliando, respectivamente: comportamento de comer compulsivo, ansiedade por se alimentar e repetição de atividades diárias. Para controlar possíveis efeitos das variáveis, sexo, idade e escolaridade, 24 participantes de G1 foram, a seguir, pareados a 24 participantes de G2 e uma nova Prova $U$ de Mann-Whitney foi realizada. Os resultados reproduziram apenas parcialmente os anteriores indicando que os itens $4,18,22,32$ e 40 não discriminaram os dois grupos.

\section{Validade de Construto}

A validade de construto foi avaliada por meio da análise fatorial exploratória, com rotação Varimax. Obteve-se 13 fatores com autovalores acima de 1,00 , sendo que os três primeiros destacavam-se dos demais com auto-valores superiores a 2,00. Verificaram-se então as comunalidades dos itens para a solução com três fatores. Os itens com maior carga no fator foram o 32 (com 78\%) e o 23 (com $72 \%$ ), e o pior item, foi o 4 (com $2 \%$ ). Os itens 4 , 17,37 e 40 não apresentaram cargas fatoriais acima de $0,30 \mathrm{em}$ nenhum dos fatores, valor usualmente considerado aceitável para sua retenção (Pasquali, 1999). Os itens 23 (Esquiva de contato sexual), 28 (Esquiva de contato físico como beijo e abraço) e 29 (Comparação da aparência física com outras pessoas ao redor) foram considerados complexos, pois apresentaram carga superior a $0,30 \mathrm{em}$ mais de um fator, ainda que as cargas mais altas se encontrassem no fator 1 (item $23=0,83$; item $28=$ 0,68 e item $29=0,57$ ). Como obtiveram $100 \%$ de concordância entre os juízes para um mesmo fator, demonstraram discriminar população clínica de não clínica na análise da validade de critério e são teoricamente relevantes para o diagnóstico do TDC, foram, todavia retidos. Os demais obtiveram carga fatorial acima de 0,53. Isto é, com covariância acima de $28 \%$ com o fator $\left(0,53^{2}\right)$, indicando que se trata de itens com alta representatividade nos respectivos fatores (Pasquali, 1999). O Fator 1 agrupou portanto 25 itens: 1, 2, 3, 5, 6, 7, 9, 10, 11, 12, $13,14,15,16,19,21,24,26,27,31,33,34,35,36$ e 39 , responsáveis por $87 \%$ da variância total. O Fator 2, que explica $3,5 \%$ da variância, ficou representado por dois itens, 18 e 32 , que se relacionam, respectivamente, a duas dimensões teoricamente diferentes, o que torna o fator não interpretável. O Fator 3, que explica $2 \%$ da variância, ficou representado por um item (22) que se refere a ansiedade por se alimentar. A Tabela 2 apresenta na primeira coluna a distribuição teórica dos 35 itens da Escala e, na segunda coluna, a distribuição dos 28 itens retidos com base nos resultados da análise fatorial. A presença de um fator dominante e a falta de justificativa teórica para os itens dos demais fatores, sugeriu a unidimensionalidade da escala. 
Tabela 2

Distribuição dos Itens com Base nos Indicadores Teóricos do TDC ( $n=35$ ), Distribuição dos Itens Retidos ( $n=28$ ) com Base em Análise Fatorial Exploratória de Componentes Principais e Descrição teórica dos Fatores com Base no DSM-IV-TR ${ }^{T M}$ (APA, 2000)

\begin{tabular}{|c|c|c|c|}
\hline Fatores & $\begin{array}{l}\text { Distribuição Teórica } \\
\text { dos itens }(n=35)\end{array}$ & $\begin{array}{l}\text { Itens Retidos } \\
\quad(n=28)\end{array}$ & $\begin{array}{l}\text { Descrição Teórica dos Fatores } \\
\text { com Base no DSM-IV-TR }{ }^{\mathrm{TM}}(\mathrm{APA}, 2000)\end{array}$ \\
\hline 1 & $1,3,7,10,13,27$ e 34 & $\begin{array}{c}1,2,3,5,6,7,9,10 \\
11,12,13,14,15,16,19 \\
21,24,26,27,31,33 \\
34,35,36 \text { e } 39\end{array}$ & $\begin{array}{l}\text { Preocupação com um imaginado defeito } \\
\text { na aparência e caso uma ligeira anomalia } \\
\text { física esteja presente, a preocupação } \\
\text { do indivíduo é acentuadamente excessiva. }\end{array}$ \\
\hline 2 & $\begin{array}{l}2,5,6,9,11,12,14 \\
15,16,18,19,21,23 \\
24,26,28,29,31,33 \\
35,36 \text { e } 39 .\end{array}$ & 18 e 32. & $\begin{array}{l}\text { A preocupação causa sofrimento } \\
\text { significativo na área clínica e prejuízo } \\
\text { no funcionamento social, ocupacional } \\
\text { e em outros campos importantes } \\
\text { da vida do indivíduo. }\end{array}$ \\
\hline 3 & $4,17,22,25,32,37$ e 40 & 22. & $\begin{array}{l}\text { Essa preocupação não é melhor } \\
\text { explicada por outros transtornos, } \\
\text { como a anorexia. }\end{array}$ \\
\hline
\end{tabular}

Tomando em conjunto os resultados da análise de conteúdo, da validade de critério e validade de construto, houve redução dos 40 itens para apenas 28 (com a eliminação dos itens: $4,8,17,18,20,22,25,30,32,37,38 \mathrm{e}$ 40). Esses reúnem em um só fator duas dimensões teóricas do TDC: a "preocupação com um imaginado defeito na aparência e caso uma ligeira anomalia física esteja presente, a preocupação do indivíduo é acentuadamente excessiva" e a "preocupação causa sofrimento significativo na área clínica e prejuízo no funcionamento social, ocupacional e em outros campos importantes da vida do indivíduo". Ou dito de forma mais sucinta, eles medem "a preocupação excessiva e o sofrimento com um imaginado defeito e os prejuizos sobre o funcionamento em diferentes áreas da vida do indivíduo". Quanto à dimensão segundo a qual "essa preocupação não é melhor explicada por outros transtornos, como a anorexia", deixou de ser representada nesta nova versão da escala.

\section{Consistência Interna}

Os 28 itens da EA-TDC revelaram excelente consistência interna (alpha de Cronbach $=0,97$ ).

\section{Considerações Finais}

A EA-TDC foi projetada para atender a necessidade de se avaliar o transtorno dismórfico corporal na área da saúde, principalmente nas áreas de cirurgia plástica, odontologia e dermatologia, pois, como citado, a maioria dos portadores de TDC é primeiramente atendida por esses profissionais. A versão original da escala, com 35 itens e três dimensões (Ramos, 2004), passou por modificações que tiveram como objetivo permitir o diag- nóstico diferencial em relação a outros transtornos que guardam relação com os sintomas do TDC (anorexia, bulimia e TOC) e também classificar a intensidade do transtorno. A versão modificada, com os 40 itens, foi então submetida à análise de construto, validade de critério e de construto, com amostras mais representativas do que a do estudo com a versão original e os resultados sugeriram uma estrutura unidimensional, composta por 28 itens, com excelente consistência interna. A EA-TDC, assim constituída, permite a avaliação da intensidade do sintoma por meio de respostas Likert segundo a qual, 0 (zero) é indicativo de nenhum sintoma, 1 (um) indicativo de sintoma leve, 2 (dois) de sintoma médio e 3 (três) de sintoma severo. A avaliação deve ser feita pela somatória dos escores atribuídos a cada um dos itens, que podem, portanto variar desde 0 a 84 . Sendo que quanto mais alto o escore, mais severos são os sintomas de TDC.

Com estas modificações a EA-TDC, que pode ser identificada como EA-TDC-28, apresenta-se como um instrumento válido para avaliar a intensidade da preocupação e o sofrimento com um defeito na aparência e prejuízo no funcionamento social, ocupacional e em outros campos importantes da vida do indivíduo. Vale lembrar, todavia, que escalas não fazem diagnósticos, permitem apenas um rastreamento do problema, fornecendo ao profissional dados objetivos do paciente. Nesse sentido, a EA-TDC deve ser empregada em situações de triagem de pacientes atendidos por profissionais da saúde, na tomada de decisão sobre a necessidade ou não de encaminhamento para atendimento psicológico ou psiquiátrico. Por se tratar de instrumento de auto-relato, de rápida aplicação, permite que já na consulta inicial seja identificada a possibilidade de ocorrência do transtorno dismórfico cor- 
poral e a necessidade de uma avaliação mais completa por um profissional da área da Psicologia ou Psiquiatria. Apesar dos resultados promissores obtidos, sugestões para futuras pesquisas incluem, a utilização de um número maior de participantes com o diagnóstico de TDC, procurando-se superar a dificuldade de acesso que usualmente se tem a essa população. Amostras maiores e formadas por extratos mais diversificados da população em termos de sexo, faixa etária, comorbidades, entre outras variáveis, deverão permitir, entre outras coisas, a definição de pontos de corte do instrumento. É preciso ainda citar a necessidade de verificar se a EA-TDC-28 permite discriminar outros transtornos que guardam relação com o TDC, uma vez que os itens desenvolvidos com essa finalidade acabaram excluídos nessa versão. A despeito dessas e de outras limitações do estudo, acredita-se que os resultados sejam relevantes para a continuidade de pesquisas que visam conhecer em mais detalhes as características do transtorno dismórfico corporal. Além do uso clínico, o instrumento poderá ser importante para fins de pesquisa na área da saúde, aumentando desta forma o número de instrumentos disponíveis para os pesquisadores.

\section{Referências}

Amaral, V. L. A. R. (2001). Transtorno dismórfico: O corpo em delírio [Resumo]. In Anais do XI Encontro Brasileiro de Psicoterapia e Medicina Comportamental (p. 35). Campinas, SP: Associação Brasileira de Psicologia e Medicina Comportamental.

American Psychiatric Association. (1980). Diagnostic statistic manual of mental disorders ( $3^{\text {rd }}$ ed.). Washington, DC: Author.

American Psychiatric Association. (1987). Diagnostic statistic manual of mental disorders ( $3^{\text {rd }}$ Rev. ed.). Washington, DC: Author.

American Psychiatric Association. (1994). Diagnostic statistic manual of mental disorders ( $4^{\text {th }} \mathrm{ed}$.). Washington, DC: Author.

American Psychiatric Association. (2000). Manual diagnóstico e estatístico de transtornos mentais - DSM-IV-TR ${ }^{T M}$ (4. ed. rev.). Porto Alegre, RS: Artmed.

Anastasi, A., \& Urbina, S. (2000). Testagem psicológica (7. ed., M. A. Veronese, Trad.). Porto Alegre, RS: Artes Médicas Sul.

Bowe, D., Leyden, J., Crerand, C., Sarwer, D., \& Margolis, D. (2007). Body dysmorphic disorder symptoms among patients with acne vulgaris. Journal of the American Academy of Dermatology, 57(2), 222-230.

Brown, T. A., Cash, T. F., \& Mikulka, P. J. (1990). Attitudinal body-image assessment: Factor analysis of the Body-Self Relations Questionnaire. Journal of Personality Assessment, 55, 135-144

Buhlmann, U., Teachman, B. A., Gerbershagen, A., Kikul, J., \& Rief, W. (2008). Implicit and explicit self-esteem and attractiveness beliefs among individuals with body dysmorphic disorder. Journal of Cognitive Therapy and Research, 32(2), 213-225.

Cooper, P. J., Taylor, M. J., Cooper, Z., \& Fairburn, C. G. (1987). The development and validation of the Body Shape Questionnaire. International Journal of Eating Disorders, 6(4), 485-494.
Dufresne, F. G., Phillips, K. A., Vittorio, C. C., \& Wilkel, C. S. (2001). A screening questionnaire for body dysmorphic disorder in a cosmetic dermatologic surgery practice. Dermatological Surgery, 27(5), 457-462.

Drummond, L. M., Pillay, A., Benson, S., \& Jones, E. (2008). Community model in treating obsessive-compulsive and body dysmorphic disorders. Psychiatric Bulletin, 32, 336-340.

Gardner, R. M. (1991). Body Dissatisfaction Scale. Journal of Youth and Adolescence, 28, 603-618.

Goodman, W. K., Price, L. H., Rasmussen, S. A., Mazure, C., Fleischmann, R. L., Hill, C. L., et al. (1989). The Yale-Brown Obsessive Compulsive Scale: Development, use, and reliability. Archives of General Psychiatry, 46, 1006-1011.

Jakubietz, M., Jakubietz, R. J., Kloss, D. F., Joerg, M. E., \& Gruenert, J. J. (2007). Body dysmorphic disorder: Diagnosis and approach. Plastic \& Reconstructive Surgery, 119(6), 1924-1930.

Moriyama, J. S. (2003). Transtorno dismórfico corporal sob a perspectiva da análise do comportamento. (Dissertação de Mestrado não-publicada). Pontifícia Universidade Católica de Campinas, SP.

Neziroglu, F. A., \& Tobias, J. A. Y. (1993). Exposure, response prevention and cognitive therapy in the treatment of body dysmorphic disorder. Behavior Therapy, 24(1), 431-438.

Pasquali, L. (1999). Instrumentos psicológicos: Manual prático de elaboração. Brasília, DF: Laboratório de Pesquisa em Avaliação e Medida.

Phillips, K. A. (1999). Body dysmorphic disorder and depression: Theorical considerations and treatment strategies. Psychiatric Quartely, 70(4), 313-331.

Phillips, K. A. (2004). Psychosis in body dysmorphic disorder. Journal of Psychiatric Research, 38(1), 63-72.

Phillips, K. A., Didie, E., \& Menard, W. (2007). Clinical features and correlates of major depressive disorder in individuals with body dysmorphic disorder. Journal of Affective Disorders, 97(3), 129-135.

Phillips, K. A., \& Kaye, W. H. (2007). The relationship of body dysmorphic disorder and eating disorders to obsessivecompulsive disorder. CNS Spectrums, 12(5), 347-358.

Phillips, K. A., McElroy, S. L., Keck, P. E., Pope, H. G., \& Hudson, J. I. (1993). Body dysmorphic disorder: 30 cases of imagined ugliness. American Journal of Psychiatry, 150(2), 302-308

Phillips, K. A., Menard, W., Pagano, M. E., Fay, C., \& Stout, R. L. (2006). Delusional versus nondelusional body dysmorphic disorder: Clinical features and course of illness. Journal of Psychiatric Research, 40(2), 95-104.

Rabinowitz, D., Neziroglu, F., \& Roberts, M. (2007). Clinical application of a behavioral model for the treatment of body dysmorphic disorder. Cognitive and Behavioral Practice, 14(2), 231-236.

Ramos, K. P. (2004). Transtorno Dismórfico Corporal: Escala para profissionais da área da saúde. (Dissertação de Mestrado não-publicada). Pontifícia Universidade Católica de Campinas, SP.

Ramos, K. P. (2009). Escala de Avaliação do Transtorno Dismórfico Corporal: Propriedades psicométricas. (Tese de Doutorado não-publicada). Pontifícia Universidade Católica de Campinas, SP.

Rohrich, R. J. (2000). The who, what, when and why of cosmetic surgery: Do our patients need a preoperative psychiatric evaluation? Plastic and Reconstructive Surgery, 106(7), 16051607. 
Rosen, J. C. (1997). Tratamiento cognitivo-conductual para el transtorno dismórfico corporal. In V. E. Caballo (Ed.), Manual para el tratamiento cognitivo-conductual de los transtornos psicologicos: transtornos por ansiedad, sexuales, afectivos y psicóticos (pp. 406-437). Madrid, España: Siglo Veintiuno.

Rosen, J. C., \& Reiter, J. (1996). Development of the body dysmorphic disorder examination. Behavioral Research Therapy, 34(9), 755-766.

Sarwer, D. B., \& Crerand, C. E. (2008). Body dysmorphic disorder and appearance enhancing medical treatments. Body Image, 5(1), 50-58.

Sarwer, D. B., Wadden, T. A., Petschuk, M. J., \& Whitaker, L. A. (1998). Body image dissatisfaction and body dysmorphic disorder in 100 cosmetic surgery patients. Plastic and Reconstructive Surgery, 101(6), 1644-1649.

Sobanski, E., \& Schimidtz, M. H. (2000). 'Everybody Looks at my public bone': A case report of an adolescent patient with body dysmorphic disorder. Acta Psychiatrica Scandinavica, 101(1), 80-82.

Warwick, H. M. C. (1995). Transtornos somatoformes y facticios. In V. E. Caballo, C. G. Buella, \& C. Carrobles (Eds.) Manual de Psicopatologia y transtornos psiquiátricos (Vol. 1, pp. 404-464). Madrid, España: Siglo Ventiuno. 